TEUBNER-TEXTE zur Informatik

Band 35

Dietmar Fey

Optik in der Rechentechnik 


\title{
TEUBNER-TEXTE zur Informatik
}

\author{
Herausgegeben von \\ Prof. Dr. Johannes Buchmann, Darmstadt \\ Prof. Dr. Udo Lipeck, Hannover \\ Prof. Dr. Franz J. Ramming, Paderborn \\ Prof. Dr. Gerd Wechsung, Jena
}

Als relativ junge Wissenschaft lebt die Informatik ganz wesentlich von aktuellen Beiträgen. Viele Ideen und Konzepte werden in Originalarbeiten, Vorlesungsskripten und Konferenzberichten behandelt und sind damit nur einem eingeschränkten Leserkreis zugänglich. Lehrbücher stehen zwar zur Verfügung, können aber wegen der schnellen Entwicklung der Wissenschaft oft nicht den neuesten Stand der Entwicklung wiedergeben.

Die Reihe TEUBNER-TEXTE zur Informatik soll ein Forum für Einzel- und Sammelbeiträge zu aktuellen Themen aus dem gesamten Bereich der Informatik sein. Gedacht ist dabei insbesondere an herausragende Dissertationen und Habilitationsschriften, spezielle Vorlesungsskripten sowie wissenschaftlich aufbereitete Abschlussberichte bedeutender Forschungsprojekte. Auf eine verständliche Darstellung der theoretischen Fundierung und der Perspektiven für Anwendungen wird besonderer Wert gelegt. Das Programm der Reihe reicht von klassischen Themen aus neuen Blickwinkeln bis hin zur Beschreibung neuartiger, noch nicht etablierter Verfahrensansätze. Dabei werden bewusst eine gewisse Vorläufigkeit und Unvollständigkeit der Stoffauswahl und Darstellung in Kauf genommen, weil so die Lebendigkeit und Originalität von Vorlesungen und Forschungsseminaren beibehalten und weitergehende Studien angeregt und erleichtert werden können.

TEUBNER-TEXTE erscheinen in deutscher oder englischer Sprache. 


\section{Dietmar Fey \\ Optik in der Rechentechnik}

Photonisches VLSI und optische Netzwerke

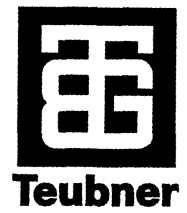

B. G. Teubner Stuttgart - Leipzig · Wiesbaden 
Die Deutsche Bibliothek - CIP-Einheitsaufnahme

Ein Titeldatensatz für diese Publikation ist bei

Der Deutschen Bibliothek erhältlich.

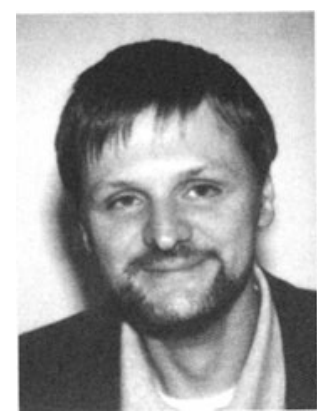

Prof. Dr.-Ing. Dietmar Fey

Geboren 1961 in Nürnberg. Von 1981 bis 1987 Studium der Informatik an der Universität Erlangen. berg mit Abschluss als Diplom-Informatiker. Von 1987 bis 1992 wissenschaftlicher Mitarbeiter am Ler für Angewandte Optik des Physikalischen Instituts der Universität Erlangen-Nürnberg. In dieser Zeit beit im Sonderforschungsbereich Multiprozessorsysteme und Netzwerkkonfigurationen. 1992 Prom 1993 Industrietätigkeit im Bereich CAD, Netzwerke und Datenbanken. Von 1994 bis 1999 wissenschaf Assistent am Institut für Informatik der Universität Jena. Dort Leiter der Arbeitsgruppe „Paralleles Opt tronisches Rechnen". 1999 Habilitation, von 1999 bis 2000 Privatdozent und Vertretung eines akadem Rates am Institut für Rechnerstrukturen der Universität-GH Siegen. Von 2000 bis 2001 Vertretunc C3-Professur an der Universität Jena. Seit 2002 Professor für Technische Informatik an der Universität

Arbeitsgebiete: Optik in der Rechentechnik, VLSI-Entwurf, Hardware/Software-Codesign, Optische werke für Cluster-Rechner.

\section{Auflage August 2002}

Alle Rechte vorbehalten

(C) B. G. Teubner GmbH, Stuttgart/Leipzig/Wiesbaden, 2002

Der Verlag Teubner ist ein Unternehmen der Fachverlagsgruppe BertelsmannSpringer. www.teubner.de

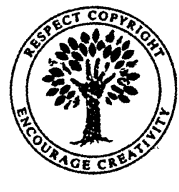

Das Werk einschließlich aller seiner Teile ist urheberrechtlich geschützt. Jede Verw $\epsilon$ außerhalb der engen Grenzen des Urheberrechtsgesetzes ist ohne Zustimmung d€ lags unzulässig und strafbar. Das gilt insbesondere für Vervielfältigungen, Überst gen, Mikroverfilmungen und die Einspeicherung und Verarbeitung in elektroni Systemen.

Die Wiedergabe von Gebrauchsnamen, Handelsnamen, Warenbezeichnungen usw. in diesem berechtigt auch ohne besondere Kennzeichnung nicht zu der Annahme, dass solche Namen im der Waren- und Markenschutz-Gesetzgebung als frei zu betrachten wären und daher von jeder benutzt werden dürften.

Umschlaggestaltung: Ulrike Weigel, www.CorporateDesignGroup.de 


\section{Vorwort}

Laut der im Auftrag der amerikanischen Regierung durchgeführten Studie "Harnessing Light - Optical Sciences and Engineering for the 21th Century" wird etwas überspitzt formuliert - das 21. Jahrhundert zum Jahrhundert der Photonik werden, wie das 20. Jahrhundert das Jahrhundert der Elektronik war. Die Photonik, die Lehre von der Physik des Lichtes sowie seiner technischen Anwendungen, prägt dabei längst unser tägliches Leben. Man denke nur an den Einsatz optischer Technologien im Informations- und Kommunikationsbereich. Nach einem Bericht der Deutschen Agenda Optische Technologien für das 21. Jahrhundert werden schon heute $90 \%$ der in Deutschland bewegten Datenmengen über das Glasfasernetz der Bundesrepublik transportiert. Der Einsatz optischer Massenspeichermedien wie CD-ROM oder DVD ist nicht mehr wegzudenken. Kürzlich angekündigte Förderprogramme des Bundesministeriums für Bildung und Forschung werden dazu beitragen, dass sich optische Technologien z.B. im Gesundheitswesen, in den Biowissenschaften, in der industriellen Fertigung und selbstverständlich auch in der Informations- und Kommunikationstechnik weiter verbreiten.

So ist es keine Vision, sondern nur eine Frage der Zeit, wann nahezu jeder Haushalt über einen Glasfaseranschluss verfügen wird. Der Siegeszug der optischen Übertragung von Daten in der Netzwerk- und Netzzugangstechnik wird auch vor der Rechentechnik nicht Halt machen, auch wenn derzeit die Optik mit Ausnahme der peripheren Speicher innerhalb der Rechner noch keine tragende Rolle innehat. Diese Einschätzung beruht auf der Tatsache, dass sich elektronische Verbindungen in der modernen Rechen- und Prozessortechnik zunehmend als der Schwachpunkt beim Streben nach höheren Leistungen erweisen. Dieses Buch zeigt auf, wie der Einsatz optischer Verbindungen in der VLSI- und Netzwerktechnik einen Ausweg aus den im Englischen mit "interconnect crisis" umschriebenen Schwierigkeiten bei den Verbindungen bietet. Dabei sei an dieser Stelle ausdrücklich betont, dass ein effizienter Einsatz optischer Verbindungen nicht ohne Einfluss auf die Architektur der Rechner und Prozessoren und der darin zum Einsatz kommenden Algorithmen bleiben kann.

Diese Themen - Technologie, Architektur und Algorithmen - sind Gegenstand des interdisziplinären Forschungsthemas "Optik in der Rechentechnik" und dieses Teubner-Textes. Das Buch verfolgt das Ziel, neben den Grundlagen auch den aktuellen Stand der Technik auf dem Gebiet der Optik in der Rechentechnik zu vermitteln. Der Erfolg des Einsatzes optischer Verbindungen in der Rechentechnik hängt entscheidend $a b$ von der interdisziplinären Zusammenarbeit zwischen Physikern, Elektrotechnikern und Informatikern. Auch dazu soll dieses 
Buch beitragen. Es versteht sich als interdisziplinäre Brücke zwischen Informatik, Optik und Elektronik. Es wendet sich an den Informatiker, der mehr über die Möglichkeiten der Optik in der Schaltkreis- und Netzwerktechnik wissen möchte, und auch an den Physiker und Ingenieur, der Genaueres über die Anwendung optischer und optoelektronischer Technologien in Architekturen erfahren will.

Forschungsinitiativen, die in der Vergangenheit auch für die "Optik in der Rechentechnik" Bedeutung hatten, waren u.a. vom Bundesministerium für Bildung und Forschung und von der Volkswagen-Stiftung geförderte Photonik-Programme. So basieren auch viele der in diesem Buch vorgestellten Themen auf Ergebnissen, die innerhalb eines von der Volkswagen-Stiftung unterstützten Forschungsprojektes "Binärer optoelektronischer Assoziativspeicher mittels SmartPixel-Technologien" in Zusammenarbeit einer Arbeitsgruppe des Autors mit Physikern und optischen Nachrichtentechnikern erzielt wurden. Ferner waren insbesondere für den in diesem Buch behandelten Themenkomplex Architektur und Algorithmen für optoelektronische Schaltkreise Ergebnisse entscheidend, die im Rahmen des von der Deutschen Forschungsgemeinschaft geförderten und vom Autor geleiteten Projekts "3-D Smart-Pixels-Rechner" gewonnen wurden. Viele der im Buch getroffenen Aussagen zur Thematik "Optische Netzwerke für Cluster-Rechner" beruhen auf Untersuchungen, die von der Arbeitsgruppe des Autors im Rahmen von Drittmittelprojekten durchgeführt wurden, die Förderung durch das Thüringer Ministerium für Wissenschaft, Forschung und Kultur und das Bundesministerium für Bildung und Forschung erhielten.

Dank sei an dieser Stelle an Prof. Dr. Werner Erhard, Universität Jena, Prof. Dr. Hartmut Bartelt, Institut für Physikalische Hochtechnologie, Jena, und Prof. Dr. Karl-Heinz Brenner, Universität Mannheim, für die Übernahme der Gutachten zu meiner Habilitationsschrift gerichtet, die eine der Grundlagen für dieses Buch bildet. Nicht vergessen bei der Danksagung möchte ich auch die Doktoranden Guido Grimm, Lutz Hoppe, Karl D. Maier, Thomas Meier von der Universität Jena und den Studenten André Flöpper von der Universität-GH Siegen, ohne deren $\mathrm{Zu}$ arbeit dieses Buch nicht zustande gekommen wäre. Dies gilt auch für Dr. Matthias Gruber und Prof. Dr. Jürgen Jahns von der FernUniversität Hagen, Dr. ErnstBernhard Kley von der Universität Jena und Dipl.-Phys. Margit Ferstl vom Heinrich-Hertz-Institut in Berlin für das Überlassen von Bildmaterial. Nicht zuletzt gilt mein Dank auch Herrn Jürgen Weiß, Leipzig, und den Herausgebern der TEUBNER-TEXTE zur Informatik, insbesondere Prof. Dr. Gerd Wechsung, ohne deren Unterstützung dieses Buch nicht entstanden wäre. Nicht zuletzt gilt mein Dank auch Sun Microsystems und dem Computerdienst Jena, die durch eine Anzeigenschaltung ebenfalls zum Zustandekommen dieses Buches beitrugen. 


\section{Inhalt}

1 Motivation und Stand der Technik.............................................................................................9

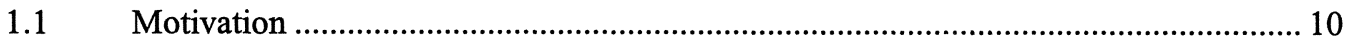

$1.2 \quad$ Entwicklung mikroelektronischer Schaltkreise ……….......................................... 11

1.2.1 Von SSI zu VLSI .................................................................................. 11

1.2.2 Die Verbindungskrise in der VLSI-Technik .................................................... 13

$1.3 \quad$ Optische Verbindungen für die Rechentechnik ........................................................... 16

1.3.1 Optische Verbindungen zwischen Baugruppen ................................................. 17

1.3.2 Optische Verbindungen auf Leiterplatten ........................................................ 20

1.3.3 Optische Verbindungen zwischen integrierten Schaltkreisen ............................... 23

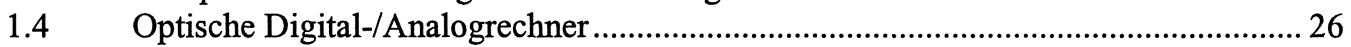

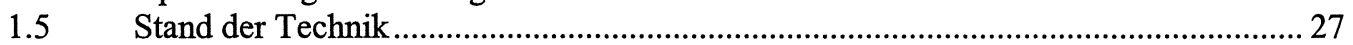

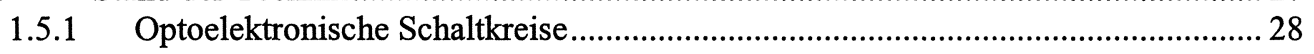

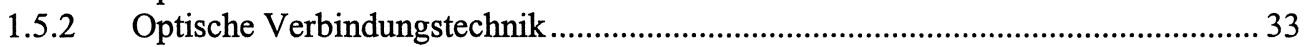

1.5.3 Optoelektronische Architekturen ...................................................................... 34

1.5.4 Entwurfswerkzeuge für 3-D OE-VLSI ......................................................... 40

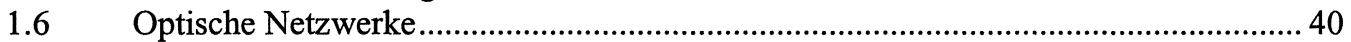

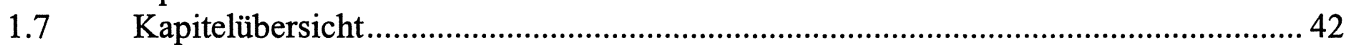

2 Einführung in die Technologie der Optik für die .........................................................................

Rechentechnik .................................................................................................................................. 44

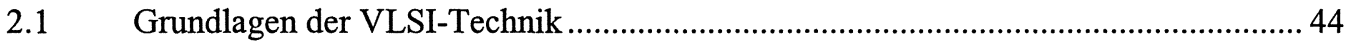

2.2 Grundlagen des CMOS-Schaltungsentwurfs ....................................................... 52

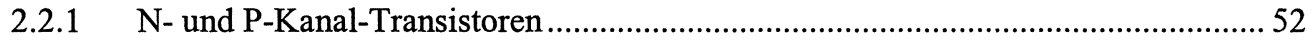

2.3 Passive optische Bauelemente zur Lichtwegeführung und -ablenkung ...................... 54

2.3.1 Diffraktive optische Elemente ………………….................................................. 55

2.3.2 Computergenerierte Elemente........................................................................... 57

2.3.3 Holographisch optische Elemente........................................................................ 63

2.3.4 Refraktive Strukturen................................................................................ 66

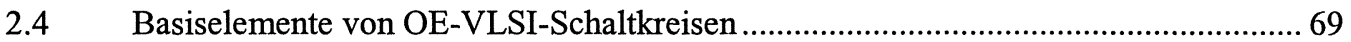

2.4.1 Physikalische Grundlagen: Absorption, spontane und stimulierte Emission.......... 70

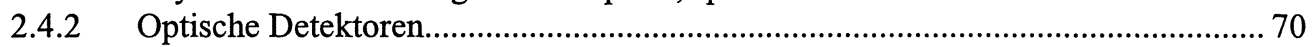

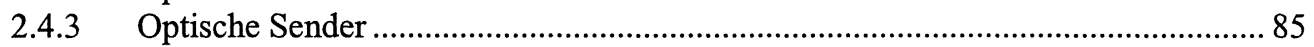

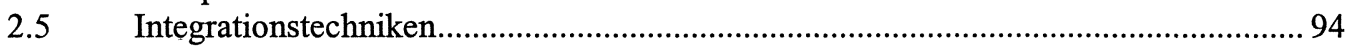

2.5.1 Gesteckte Optik............................................................................................... 95

2.5.2 Planare Optik .............................................................................................. 96

2.5.3 Faserfelder …………………………………........................................ 99

3 Allgemeine Leistungsanalyse von 3-D OE-VLSI-Architekturen ....................................... 102

3.1 Abstraktes Architekturmodell für 3-D OE-VLSI-Systeme........................................ 103

3.2 Mathematische Formeln für die parametrisierte Leistungsanalyse.............................. 106 
4 Architekturbeispiele für effiziente 3-D OE-VLSI-Schaltkreise .................................. 113

4.1 Ein optoelektronischer superskalarer 3-D Prozessor für Ganzzahlarithmetik ........... 114

4.1.1 Vorzeichenbehaftete Zahlendarstellung....................................................... 115

4.1.2 Addition und Subtraktion........................................................................... 116

4.1.3 Realisierung der Multiplikation ................................................................. 117

4.1.4 Realisierung der Division ............................................................................ 118

4.1.5 Rückkonvertierung einer SD-Zahl ins 2er-Komplement ................................... 120

4.1.6 Abbildung auf eine optoelektronische 3-D Architektur ....................................... 121

4.1.7 Abschätzung der Rechenleistung .................................................................. 128

4.1.8 Erste Realisierung und Simulationsergebnisse ............................................ 132

4.2 Optoelektronische 3-D Prozessoren für Festpunktarithmetik ................................ 134

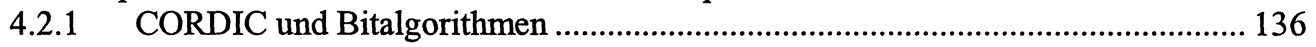

4.2.2 Der verallgemeinerte CORDIC................................................................. 140

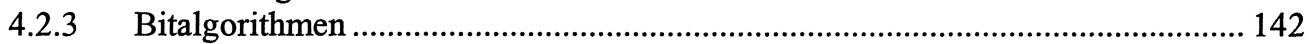

4.2.4 Abbildung auf eine 3-D OE-VLSI-Architektur ................................................. 146

4.3 Optisch rekonfigurierbare Hardware ............................................................... 154

4.3.1 Prinzipielle Vorteile optischer Verbindungen für dynamisch rekonfigurierbare

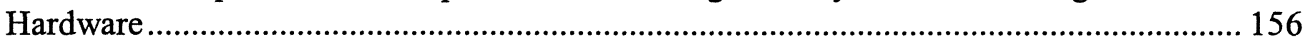

4.3.2 Beispiele für optoelektronisch rekonfigurierbare Hardware ............................... 159

4.3.3 OptoRAP - ein Konzept für eine dynamische rekonfigurierbare optoelektronische

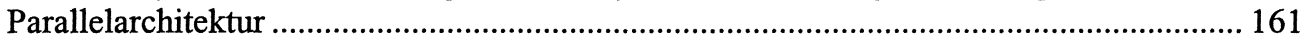

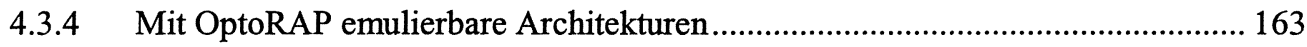

4.4 Ein optoelektronischer paralleler Bildverarbeitungsprozessor für Binärbilder.......... 165

4.4.1 Die Operationen des Bildverarbeitungsprozessors .......................................... 167

4.4.2 Architektur des Prozessorelements ................................................................... 176

4.4.3 Simulation und Logiksynthese des optischen Bildverarbeitungsprozessors ......... 182

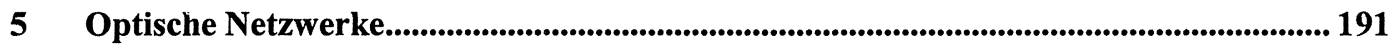

5.1 Physikalische Grundlagen optischer Netzwerke................................................. 192

5.2 Optischer Wellenlängenmultiplex ...................................................................... 198

5.3 Komponenten eines optischen WDM-Netzwerkes ................................................ 200

5.4 Architekturen optischer Netze ............................................................................. 207

5.5 Routing-Verfahren in WDM-Ring-Netzen ....................................................... 211

Literaturverzeichnis .............................................................................................................................. 215

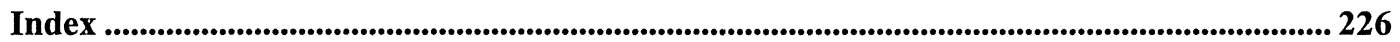

